\title{
Florística vascular não arbórea em uma Reserva Particular do Patrimônio Natural (RPPN) na Floresta Atlântica de São Paulo, Brasil ${ }^{1}$
}

Non-arboreal vascular flora of an Atlantic Forest area in Sao Paulo, Brazil

\author{
Mariana Naomi Saka ${ }^{2,3}$ \& Julio Antonio Lombardi ${ }^{2}$
}

\begin{abstract}
Resumo
A Reserva Particular do Patrimônio Natural (RPPN) Paiol Maria possui 76 ha deFloresta Ombrófila Densa Montana e está localizada no município de São Lourenço da Serra, São Paulo, Brasil, pertencente ao Corredor de Biodiversidade da Serra do Mar. Objetivando elaborar uma listagem florística das espécies vasculares não arbóreas ocorrentes na RPPN, foram realizadas coletas durante o período de junho de 2010 a setembro de 2011 . Foram inventariadas 330 espécies, distribuídas em 84 famílias e 223 gêneros. Das espécies amostradas, quatro são consideradas ameaçadas no Estado de São Paulo. A análise de similaridade entre as espécies epífitas ocorrentes na RPPN e outras quatro áreas de Floresta Ombrófila Densa Montana localizadas na Serra do Mar reafirma a importância de fragmentos em regeneração secundária para o conhecimento e conservação da biodiversidade da Floresta Atlântica.

Palavras-chave: floresta montana, floresta secundária, Floresta Atlântica, epífitas.
\end{abstract}

\begin{abstract}
The private owned conservation area of RPPN Paiol Maria includes 76 ha of montane forest located at the municipality of São Lourenço da Serra, São Paulo State, belongs to the biodiversity corridor of the Serra do Mar, and is located at the northern part of Ribeira valley. This study was carried out during the period of June 2010 to September 2011 and our aim was to elaborate a checklist of the non-arboreal species occurring in the RPPN. A total of 330 species, distributed in 84 families and 223 genera were collected. Four species recorded for this work appears in the red list for the state of São Paulo. A similarity analysis between the epiphytes found in the RPPN and other four areas of montane forest reassert the important role of secondary forest fragments to the knowledge and conservation of the Atlantic Forest biodiversity.
\end{abstract}

Key words: montane forest, secondary forest, Atlantic Forest, epiphytes.

\section{Introdução}

A extensão original da Floresta Atlântica, estimada em 1-1,5 milhões de $\mathrm{km}^{2}$ (Galindo-Leal \& Câmara 2005), atualmente tem área remanescente de cerca de $11,7 \%$, restrita principalmente a unidades de conservação ou fragmentos com menos de 50 ha (Ribeiro et al. 2009). Os amplos gradientes latitudinal $\left(3-30^{\circ} \mathrm{S}\right)$, altitudinal (nível do mar a $2.700 \mathrm{~m}$ ), e longitudinal (da costa brasileira ao leste do Paraguai e Argentina), além de proporcionarem heterogeneidade à sua composição, que abrange formações vegetacionais tropicais a subtropicais (Tabarelli et al. 2010), também propiciam alta diversidade e endemismo de espécies animais e vegetais (Silva \& Casteletti 2005). Devido à grande pressão de fragmentação e alto número de espécies endêmicas, a Floresta Atlântica é considerada um dos 34 hotspots de diversidade mundial (Mittermeier et al. 2005).

A Floresta da Serra do Mar, que se estende do Rio de Janeiro ao Paraná, é o maior remanescente de Floresta Atlântica no Brasil (Ribeiro et al. 2009). Em conjunto com as matas do nordeste do Brasil, abriga cerca de $85 \%$ das espécies endêmicas do bioma (Werneck et al. 2011). É considerada um importante centro de biodiversidade (Aguiar et al. 2003), e concentra grande riqueza genética (Carnaval et al. 2009). A região da Serra do Mar

\footnotetext{
${ }^{1}$ Dissertação apresentada para obtenção do título de Mestre em Biologia Vegetal pelo Programa de Pós-graduação em Biologia Vegetal do Instituto de Biociências de Rio Claro.

${ }^{2}$ Universidade Estadual Paulista - UNESP, Inst. Biociências de Rio Claro, Depto. Botânica, Av. 24A 1515, 13506-900, Rio Claro, SP, Brasil.

${ }^{3}$ Autor para correspondência: marianasaka.bio@gmail.com
} 
possui cerca de $25 \%$ de sua área enquadrada em unidades de conservação (Ribeiro et al. 2009). Entretanto, outros fragmentos com menos de 200 ha podem contribuir para reduzir o isolamento entre fragmentos maiores funcionando como corredores de biodiversidade (Morellato \& Haddad 2000; Ribeiro et al. 2009).

A bacia do rio Ribeira de Iguape, região também denominada Vale do Ribeira, está delimitada pelas Serras do Mar, de Paranapiacaba e de Mongaguá (São Paulo 1966). Esta região, em parte devido a características naturais, sobretudo o relevo, manteve-se à margem do desenvolvimento observado no restante do Estado de São Paulo (Pavan-Fruehalf 2000). A região é rica em mananciais e, por isso, parcelas significativas de remanescentes do Vale do Ribeira estão enquadradas em diversos tipos de unidades de conservação (Hogan et al. 2000), como a Reserva Particular do Patrimônio Natural (RPPN) Paiol Maria, objeto deste estudo.

Em sua maioria, os fragmentos remanescentes da Floresta Atlântica são constituídos por formações secundárias em diferentes estágios sucessionais (Morellato \& Haddad 2000) e sua dinâmica e conservação tem sido alvo de estudos no Brasil (e.g. Oliveira-Filho et al. 2004; Liebsch et al. 2007).

$\mathrm{O}$ estrato não arbóreo pode representar cerca de $80 \%$ da diversidade de espécies em florestas (Gentry 1992) e, em florestas de regeneração secundária, são potencialmente determinantes ao contribuir para a ciclagem de nutrientes essenciais e influenciar a composição de espécies de uma floresta regenerante (Gilliam 2007). Devido à especificidade de recursos e habitat, plantas raras do estrato herbáceo podem ser utilizadas como indicadoras de conservação e diversidade (Spyreas \& Matthews 2006). Menini Neto et al. (2009) avaliaram o potencial da composição florística de espécies epífitas como indicadora de estado de conservação em fragmentos do sudeste do estado de Minas Gerais e chegaram à conclusão de que é importante conservar qualquer tipo de fragmento, independente de área ou tipo de vegetação.

O objetivo deste trabalho foi elaborar uma listagem florística das espécies vasculares não arbóreas ocorrentes na Reserva Particular do Patrimônio Natural (RPPN) Paiol Maria, e conduzir uma análise de similaridade da composição de espécies epífitas entre a área da RPPN e quatro áreas de Floresta Ombrófila Densa Montana pertencentes ao corredor de biodiversidade da Serra do Mar, contribuindo para o conhecimento do estrato não-arbóreo em florestas secundárias no Estado de São Paulo.

\section{Material e Métodos}

Área de estudo

O município de São Lourenço da Serra é o primeiro, a partir da capital, que integra a região do Vale do Ribeira. Enquadra-se na microrregião de Itapecerica da Serra e na mesorregião da cidade de São Paulo (IBGE 2002), Brasil, e está localizado a $23^{\circ} 51^{\prime} 02^{\prime}$ 'S e $46^{\circ} 36^{\prime} 56^{\prime \prime}$ O (Fig. 1). O clima, segundo a classificação de Köppen, é Cwa, ou seja, temperado úmido com verão quente e inverno seco, a temperatura anual oscilando entre a mínima do mês mais frio de $9,4^{\circ} \mathrm{C}$ e a máxima do mês mais quente de $28,8^{\circ} \mathrm{C}$, e a precipitação média anual de $2400 \mathrm{~mm}$ a $2700 \mathrm{~mm}$ (CEPAGRI 2011).

A Reserva Particular do Patrimônio Natural (RPPN) Paiol Maria abrange 76 ha dos 240,8 ha do Sítio Paiol Maria (altitude de 700 a $836 \mathrm{~m}$ ). A área pertence à Ordem dos Servos de Maria, porém, desde 2005, é administrada pela ONG "Vitae Civilis - Instituto para o Desenvolvimento, Meio Ambiente e Paz" em regime de comodato. Entre 2006 e 2007, a ONG, o Ministério do Meio Ambiente (PDA

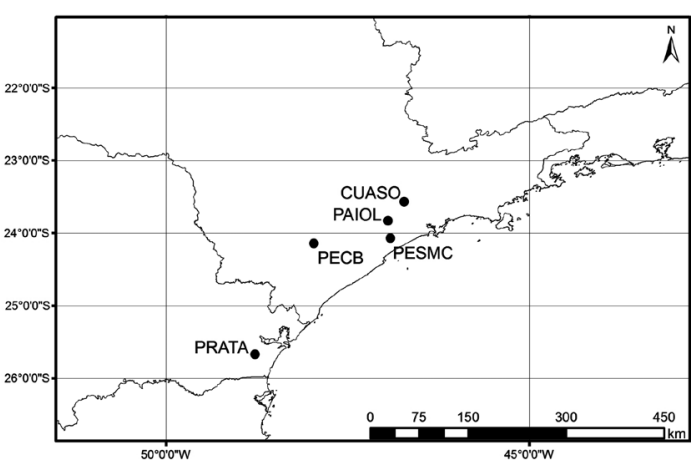

Figura 1 - Localização da Reserva Particular do Patrimônio Natural (RPPN) Paiol Maria e das áreas utilizadas na análise de similaridade florística. CUASO (Reserva da Cidade Universitária Armando Salles de Oliveira, SP), PAIOL (RPPN Paiol Maria, SP), PECB (Parque Estadual Carlos Botelho, SP), PESMC (Parque Estadual da Serra do Mar, Núcleo Curucutu, SP), PRATA (Serra da Prata, PR).

Figure 1 - Location of the Reserva Particular do Patrimônio Natural (RPPN) Paiol Maria, and of the other areas used in floristic similarity analysis. CUASO (Reserva da Cidade Universitária Armando Salles de Oliveira, SP), PAIOL (RPPN Paiol Maria, SP), PECB (Parque Estadual Carlos Botelho, SP), PESMC (Parque Estadual da Serra do Mar, Núcleo Curucutu, SP), PRATA (Serra da Prata, PR). 
Tabela 1 - Área, altitude e local dos levantamentos utilizados na análise de similaridade florística considerando espécies epífitas. Table 1 - Area, elevation and localization of the surveys used in floristic similarity analysis considering the epiphytic species.

\begin{tabular}{|c|c|c|c|c|c|}
\hline Sigla & Local & Altitude & $\begin{array}{l}\text { Área } \\
\text { (ha) }\end{array}$ & $\begin{array}{c}\text { Número } \\
\text { de espécies } \\
\text { epífitas }\end{array}$ & Referência \\
\hline CUASO & $\begin{array}{l}\text { Reserva da Cidade Universitária } \\
\text { Armando Salles de Oliveira (SP) }\end{array}$ & $735-770$ & 10 & 34 & $\begin{array}{l}\text { Dislich \& Mantovani } \\
\text { (1998) } \\
\text { Groppo \& Pirani (2005) }\end{array}$ \\
\hline PAIOL & RPPN Paiol Maria (SP) & $700-836$ & 76 & 74 & Presente trabalho \\
\hline PECB & $\begin{array}{l}\text { Parque Estadual de Carlos } \\
\text { Botelho (SP) }\end{array}$ & $30-1000$ & 37644 & 262 & Lima et al. 2011 \\
\hline PESMC & $\begin{array}{l}\text { Parque Estadual da Serra do Mar } \\
\text { - Núcleo Curucutu (SP) }\end{array}$ & $750-850$ & 12090 & 47 & Garcia \& Pirani (2005) \\
\hline PRATA & Serra da Prata (PR) & $500-1100$ & $25000^{*}$ & 250 & Blum et al. (2011) \\
\hline
\end{tabular}

Número de espécies epífitas refere-se àquelas identificadas a nível de espécie, *área do Parque Nacional Saint-Hilaire \& Lange.

Number of epiphytic species refers to the ones identified at species level, *Saint-Hilaire \& Lange National Park area.

Mata Atlântica) e a Petrobrás, investiram em infraestrutura para um projeto ecoturístico na área, que compreendeu a demarcação de trilhas para visitação e construção de um alojamento (Neiman et al.2009; Vitae Civilis 2012). Sua vegetação é classificada como Floresta Ombrófila Densa Montana (FODM) (IBGE 2012), e como toda a região adjacente, a área do Sítio Paiol Maria foi utilizada na extração de carvão vegetal, entre o final do século XIX e início do século XX. Desta maneira, a área apresenta alguns pontos em regeneração avançada e outros pontos menos preservados, onde pode ser verificada interferência humana, como a presença de uma torre de alta tensão que corta a área da RPPN, e resquícios de construções, além de remanescentes de cultivo de plantas não nativas da flora brasileira.

\section{Levantamento florístico}

Foram realizadas 10 expedições de coleta de material botânico, de junho de 2010 a setembro de 2011, num período de dois a três dias por vez. A amostragem foi efetuada através do método de caminhamento (Filgueiras et al. 1994), percorrendo-se trilhas já demarcadas a partir do projeto ecoturístico no Paiol Maria pela "Vitae Civilis" e ocasionais incursões perpendiculares a esses caminhos. Foram coletados espécimes em estado fértil de plantas vasculares não classificadas como árvores de acordo com Mori et al. (1989). O material herborizado foi incorporado ao Herbário Rioclarense (HRCB) do Instituto de Biociências da Universidade Estadual Paulista, campus de Rio Claro.
Para análise e a identificação do material, foram utilizadas bibliografia específica, comparação e consulta a especialistas. As famílias de Angiospermas seguem a proposta da APG III (2009), enquanto aquelas das licófitas e monilófitas estão baseadas, respectivamente, em Kramer \& Tryon (1990) e Smith et al. (2006). Todas as espécies foram classificadas de acordo com o hábito (Richards 1996) e forma de vida (Mori et al. 1989). As espécies não nativas da flora brasileira foram assim consideradas em consulta na BFG (2015) e as espécies consideradas ameaçadas estão de acordo com o Livro Vermelho das Espécies Vegetais Ameaçadas do Estado de São Paulo (Mamede et al. 2007).

A comparação da composição de espécies de epífitas foi realizada com outros levantamentos em áreas de FODM pertencentes ao corredor de biodiversidade da Serra do Mar: Parque Estadual Carlos Botelho (Lima et al. 2011), Parque Estadual da Serra do Mar - Núcleo Curucutu (Garcia \& Pirani 2005), Reserva da Cidade Universitária Armando Salles de Oliveira (Dislich \& Mantovani 1998; Groppo \& Pirani 2005) e Serra da Prata, parte norte do Parque Nacional Saint-Hilaire \& Lange (Blum et al. 2011) (Fig. 1; Tab. 1). A flora de epífitas foi escolhida para a análise de similaridade florística pois além de serem potenciais indicadores de conservação (Menini Neto et al. 2009), não existem muitos levantamentos gerais ou que foquem a flora não arbórea para áreas de FODM. Foi calculado o Índice de Similaridade de Jaccard (MuellerDombois \& Ellenberg 1974) e efetuada posterior 
análise de agrupamento (UPGMA) através do programa FITOPAC 2.1 (Shepherd 2009). No levantamento de Blum et al. (2011), as espécies consideradas epífitas acidentais foram excluídas e em todas as listagens foi realizado processo de exclusão de táxons não determinados e adequação da nomenclatura de acordo com BFG (2015).

\section{Resultados e Discussão}

Foram coletadas 330 espécies, distribuídas em 84 famílias (Tab. 2), sendo 18 famílias e 56 espécies de samambaias e licófitas, e 66 famílias e 274 espécies de angiospermas. Três espécies (Aechmea gracilis, Nematanthus monanthos e Zygopetalum maxillare) estão presentes na Lista de Espécies Ameaçadas do Estado de São Paulo como vulneráveis e uma espécie (Psychotria racemosa) como presumivelmente extinta. Apenas 10 espécies não são nativas da flora brasileira.

O índice de similaridade de espécies epífitas entre a RPPN Paiol Maria e os demais levantamentos de FODM foi cerca de 10\%. A composição de espécies epífitas da RPPN Paiol Maria é mais próxima das áreas do Parque Estadual de Carlos Botelho (PECB) e da Serra da Prata e da área do Núcleo Curucutu do Parque Estadual da Serra do Mar (Fig. 2). As áreas do PECB e da Serra da Prata apresentam 30\% de similaridade, o que pode ter ocorrido pelo maior número de espécies epífitas listadas para as duas áreas, diretamente proporcional ao tamanho da área estudada e do tipo de levantamento realizado. Enquanto que Blum et al. (2011) focaram somente nas espécies epífitas, em Lima et al. (2011) todos os estratos foram amostrados e coletas de trabalhos anteriores também foram incluídas. Entretanto, os valores dos índices de similaridade não são significativos e, assim como em Menini Neto et al. (2009), a proximidade das áreas não implicou na similaridade entre as floras epifíticas, o que ressalta a importância da conservação de cada fragmento.

Mesmo em fragmentos pequenos em áreas com vegetação relativamente bem conhecida, como é o caso do trecho paulista da Serra do Mar, pode-se descobrir a ocorrência de espécies ainda não registradas para o Estado, como o caso de Asterostigma luschnatianum, cujo primeiro registro para o Estado de São Paulo (Gonçalves 2012) foi feito a partir de coletas realizadas neste estudo. Desta maneira, existe a necessidade de levantamentos que enfoquem também a flora herbácea e arbustiva, uma vez que existem poucos trabalhos que abrangem a flora não arbórea e, dentre estes, os mais comuns são aqueles que abordam a flora epifítica.

Áreas de regeneração secundária tem maior probabilidade de abrigarem grande riqueza de espécies ao se encontrarem próximas a populações de áreas de vegetação primária ou

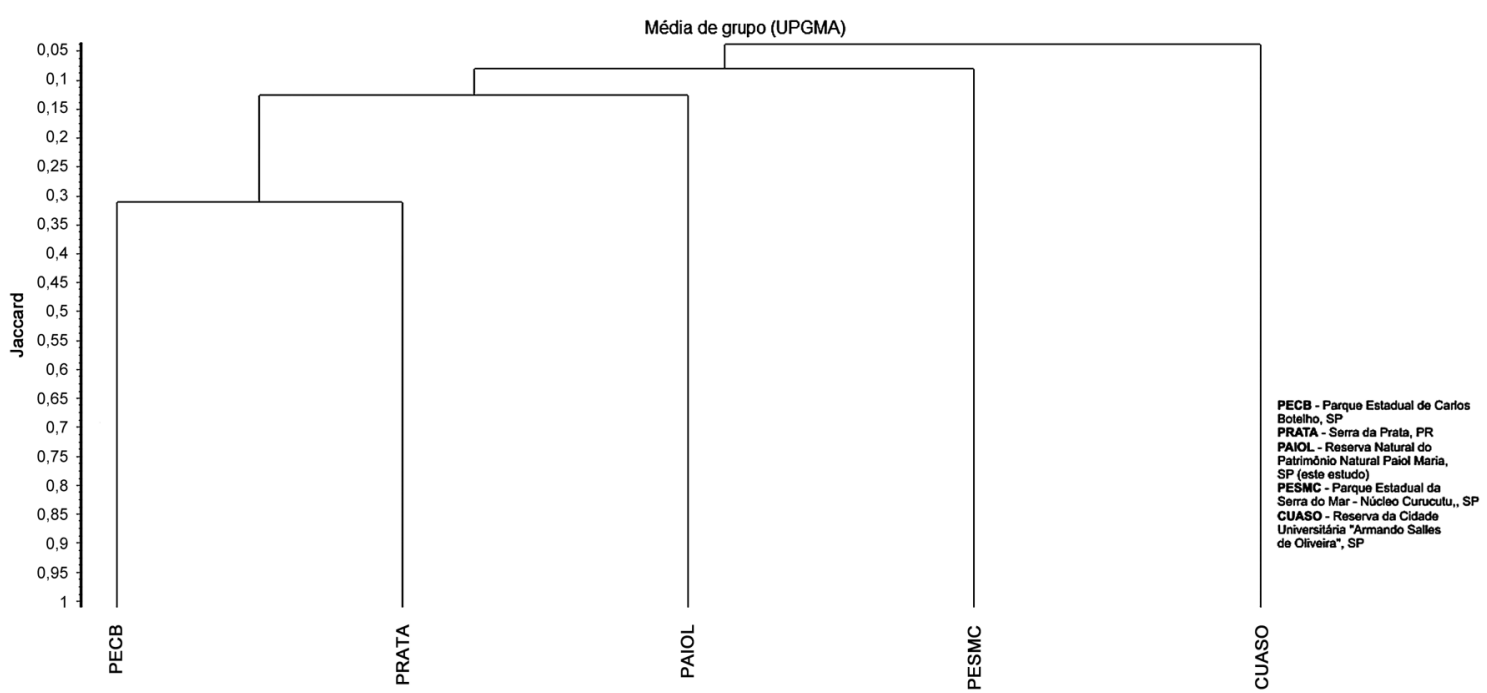

Figura 2 - Índice de similaridade (coeficiente de Jaccard) da composição de espécies epífitas entre a Reserva Particular do Patrimônio Natural (RPPN) Paiol Maria e as demais áreas analisadas.

Figure 2 - Similarity index (Jaccard's coefficient) obtained by the composition of epiphytic species occurring at the Reserva Particular do Patrimônio Natural (RPPN) Paiol Maria and other areas analyzed. 
protegidas (Lees \& Peres 2008). A área de estudo, por se localizar no Corredor de Biodiversidade da Serra do Mar, pode ser complementar à conservação de áreas protegidas geograficamente próximas (Barlow et al. 2007), ao ter o potencial de mitigar extinções causadas pelo declínio de áreas com vegetação primária (Wright \& MullerLandau 2006), e funcionar como refúgio crítico para espécies nativas remanescentes em áreas com alta pressão antrópica (Chazdón et al. 2009).

Tabela 2 - Famílias e espécies de plantas vasculares não arbóreas registradas para a RPPN Paiol Maria, São Lourenço da Serra, SP. Categoria de ameaça de acordo com Mamede et al. (2007).

Table 2 - Non-arboreal vascular plants families and species recorded in RPPN Paiol Maria, São Lourenço da Serra, SP. Threatened category follows Mamede et al. (2007).

\begin{tabular}{|c|c|c|}
\hline Família/ Espécies & $\begin{array}{l}\text { Forma de vida, Hábito, } \\
\text { Categoria de ameaça }\end{array}$ & Coletor $n^{\circ}$ \\
\hline \multicolumn{3}{|l|}{ LICÓFITAS } \\
\hline \multicolumn{3}{|l|}{ LYCOPODIACEAE (A.Salino ) } \\
\hline Palhinhaea cernua (L.) Franco \& Vasc. & $\mathrm{Er}, \mathrm{Te}, \mathrm{NT}$ & JAL 7875 \\
\hline \multicolumn{3}{|l|}{ SELAGINELLACEAE (A.Salino) } \\
\hline Selaginella decomposita Spring & Er, Te, NT & JAL 7853 \\
\hline \multicolumn{3}{|l|}{ SAMAMBAIAS } \\
\hline \multicolumn{3}{|l|}{ ANEMIACEAE } \\
\hline Anemia phyllitidis (L.) Sw. & $\mathrm{Er}, \mathrm{Te}, \mathrm{NT}$ & MNS 31 \\
\hline \multicolumn{3}{|l|}{ ASPLENIACEAE (A.Salino) } \\
\hline Asplenium harpeodes Kunze & Er, Ep, NT & JAL 8052 \\
\hline Asplenium mucronatum C.Presl & Er, Ep, NT & MNS 99 \\
\hline Asplenium scandicinum Kaulf. & Er, Ep, NT & JAL 7810 \\
\hline \multicolumn{3}{|l|}{ ATHYRIACEAE (A.Salino) } \\
\hline Diplazium ambiguum Raddi & Er, Te, NT & JAL 7884 \\
\hline Diplazium plantaginifolium (L.) Urb. & $\mathrm{Er}, \mathrm{Te}, \mathrm{NT}$ & JAL 7877 \\
\hline \multicolumn{3}{|l|}{ BLECHNACEAE (A.Salino) } \\
\hline Blechnum brasiliense Desv. & $\mathrm{Er}, \mathrm{Te}, \mathrm{NT}$ & MNS 33, 126 \\
\hline Blechnum cordatum (Desv.) Hieron. & $\mathrm{Er}, \mathrm{Te}, \mathrm{NT}$ & JAL 8056 \\
\hline Blechnum polypodioides Raddi & $\mathrm{Er}, \mathrm{Te}, \mathrm{NT}$ & MNS 129 \\
\hline Salpichlaena volubilis (Kaulf.) J.Sm. & Er, Tr, NT & JAL 7796 \\
\hline \multicolumn{3}{|l|}{ CYATHEACEAE (A.Salino) } \\
\hline Alsophila setosa Kaulf. & Arb, Te, NT & JAL 8059 \\
\hline Cyathea atrovirens (Langsd. \& Fisch.) Domin & Arb, Te, NT & JAL 7867, 7966 \\
\hline Cyathea corcovadensis (Raddi) Domin & Arb, Te, NT & JAL 7797 \\
\hline Cyathea phalerata Mart. & Arb, Te, NT & JAL 8046 \\
\hline \multicolumn{3}{|l|}{ DENNSTAEDTIACEAE (F.C.Assis \& A.Salino) } \\
\hline Dennstaedtia dissecta (Sw.) T.Moore & $\mathrm{Er}, \mathrm{Te}, \mathrm{NT}$ & JAL 8522, 7964 \\
\hline Hypolepis stolonifera var. nebularis Schwartsb. & $\mathrm{Er}, \mathrm{Te}, \mathrm{NT}$ & JAL 7868 \\
\hline Pteridium arachnoideum (Kaulf.) Maxon & Er, Te, NT & JAL 7843 \\
\hline \multicolumn{3}{|l|}{ DRYOPTERIDACEAE (A.Salino) } \\
\hline Didymochlaena truncatula (Sw.) J.Sm. & $\mathrm{Er}, \mathrm{Te}, \mathrm{NT}$ & JAL 7805 \\
\hline
\end{tabular}




\begin{tabular}{|c|c|c|}
\hline Família/ Espécies & $\begin{array}{l}\text { Forma de vida, Hábito, } \\
\text { Categoria de ameaça }\end{array}$ & Coletor $n^{\circ}$ \\
\hline Elaphoglossum glaziovii (Fée) Brade & Er, Ep, NT & JAL 7842 \\
\hline Elaphoglossum lingua (C.Presl) Brack & Er, Ep, NT & JAL 7934 \\
\hline Lastreopsis amplissima (C.Presl) Tindale & Er, Te, NT & MNS 30 \\
\hline Megalastrum connexum (Kaulf.) A.R. Sm. \& R.C. Moran & Er, Te, NT & MNS80 \\
\hline Polybotrya cylindrica Kaulf. & Er, Ep, NT & JAL 7848 \\
\hline Polybotrya semipinnata Fée & Er, Ep, NT & JAL 7904 \\
\hline \multicolumn{3}{|l|}{ GLEICHENIACEAE (T.E.Almeida) } \\
\hline Sticherus bifidus (Willd.) Ching & Er, Te, NT & JAL 7873 \\
\hline \multicolumn{3}{|l|}{ HYMENOPHYLLACEAE (A.Salino \& T.E.Almeida) } \\
\hline Didymoglossum reptans (Sw.) C.Presl & Er, Ep, NT & JAL 8529 \\
\hline Polyphlebium angustatum (Carmich.) Ebihara \& Dubuisson & Er, Ep, NT & JAL 7788 \\
\hline Trichomanes elegans Rich. & Er, Ep, NT & JAL 7939 \\
\hline Trichomanes polypodioides $\mathrm{L}$. & Er, Ep, NT & JAL7812, 8540 \\
\hline Vandenboschia collariata (Bosch) Ebihara \& Dubuisson & Er, Ep, NT & JAL 7814 \\
\hline \multicolumn{3}{|l|}{ LINDSAEACEAE (A.Salino) } \\
\hline Lindsaea botrychioides A.St.Hil. & $\mathrm{Er}, \mathrm{Te}, \mathrm{NT}$ & JAL 7847 \\
\hline Lindsaea quadrangularis Raddi subsp. quadrangularis & Er, Te, NT & JAL 7794 \\
\hline Lindsaea virescens $\mathrm{Sw}$. & $\mathrm{Er}, \mathrm{Te}, \mathrm{NT}$ & JAL 7809 \\
\hline \multicolumn{3}{|l|}{ LOMARIOPSIDACEAE (A.Salino) } \\
\hline Nephrolepis brownii (Desv.) Hovenkamp \& Miyam.* & $\mathrm{Er}, \mathrm{Te}$ & JAL 7844 \\
\hline \multicolumn{3}{|l|}{ LYGODIACEAE } \\
\hline Lygodium volubile Sw. & $\mathrm{Er}, \mathrm{Tr}, \mathrm{NT}$ & JAL 8055 \\
\hline \multicolumn{3}{|l|}{ MARATTIACEAE (A.Salino) } \\
\hline Danaea geniculata Raddi & $\mathrm{Er}, \mathrm{Te}, \mathrm{NT}$ & JAL 7917 \\
\hline Danaea moritziana C.Presl & $\mathrm{Er}, \mathrm{Te}, \mathrm{NT}$ & JAL 8512 \\
\hline Marattia cicutifolia Kaulf. & $\mathrm{Er}, \mathrm{Te}, \mathrm{NT}$ & JAL 7804 \\
\hline \multicolumn{3}{|l|}{ POLYPODIACEAE (A.Salino, T.E.Almeida \& F.C.Assis) } \\
\hline Cochlidium punctatum (Raddi) L.E.Bishop & $\mathrm{Er}, \mathrm{Te}, \mathrm{NT}$ & JAL 8504 \\
\hline Microgramma squamulosa (Kaulf.) de la Sota & Er, Ep, NT & MNS 36, 111 \\
\hline Microgramma cf. vaccinniifolia (Langsd. \& Fisch.) Copel. & Er, Ep, NT & MNS 127 \\
\hline Niphidium crassifolium (L.) Lellinger & Er, Ep, NT & JAL 8636 \\
\hline Pecluma recurvata (Kaulf.) M.G.Price & Er, Ep, NT & JAL 7889 \\
\hline Pecluma robusta (Fée) M. Kessler \& A.R.Sm. & Er, Ep, NT & JAL 7795 \\
\hline Pecluma truncorum (Lindm.) M.G.Price & Er, Ep, NT & JAL 7893 \\
\hline Pleopeltis hirsutissima (Raddi) de la Sota & Er, Ep, NT & JAL 7787,7874 \\
\hline Serpocaulon catharinae (Langsd. \& Fisch.) A.R.Sm. & Er, Ep, NT & JAL 8037 \\
\hline Serpocaulon fraxinifolium (Jacq.) A.R.Sm. & Er, Ep, NT & MNS 110, 130 \\
\hline \multicolumn{3}{|l|}{ PTERIDACEAE (A.Salino \& T.E. Almeida) } \\
\hline Doryopteris lomariacea Klotzsch & $\mathrm{Er}, \mathrm{Te}, \mathrm{NT}$ & MNS 109 \\
\hline Pteris decurrens C.Presl & $\mathrm{Er}, \mathrm{Te}, \mathrm{NT}$ & MNS 32,94 \\
\hline
\end{tabular}




\begin{tabular}{|c|c|c|}
\hline Família/ Espécies & $\begin{array}{l}\text { Forma de vida, Hábito, } \\
\text { Categoria de ameaça }\end{array}$ & Coletor $\mathbf{n}^{\circ}$ \\
\hline Vittaria lineata (L.) Sm. & Ep, Te, NT & JAL 8071 \\
\hline \multicolumn{3}{|l|}{ THELYPTERIDACEAE (A.Salino) } \\
\hline Thelypteris amambayensis (Christ.) Ponce & Er, Te, NT & JAL 7883 \\
\hline Thelypteris decurtata (Link) de la Sota & Er, Te, NT & JAL 8061 \\
\hline Thelypteris regnelliana (C.Chr.) Ponce & Er, Te, NT & JAL 7876, 7927 \\
\hline \multicolumn{3}{|l|}{ ANGIOSPERMAS } \\
\hline \multicolumn{3}{|l|}{ ACANTHACEAE } \\
\hline Hygrophila costata Nees & $\mathrm{Er}, \mathrm{Te}, \mathrm{NT}$ & JAL 8063 \\
\hline Mendoncia velloziana Mart. & Arb, Tr, NT & JAL 8521 \\
\hline Pachystachys sp. & $\mathrm{Er}, \mathrm{Te}$ & JAL 8043 \\
\hline \multicolumn{3}{|l|}{ ALISMATACEAE } \\
\hline Echinodorus grandiflorus (Cham. \& Schltdl.) Michelli & $\mathrm{Er}, \mathrm{Aq}, \mathrm{NT}$ & JAL 8065 \\
\hline \multicolumn{3}{|l|}{ AMARANTHACEAE } \\
\hline Althernanthera brasiliana (L.) Kuntze & Arb, Te, NT & MNS 42 \\
\hline Celosia grandifolia Moq. & Er, Te, NT & MNS 98 \\
\hline Chamissoa acuminata Mart. var. acuminata & Er, Te, NT & JAL 7948,8505 \\
\hline Hebanthe eriantha (Poir.) Pedersen & Er, Te, NT & MNS114 \\
\hline Iresine diffusa Humb. \& Bonpl. ex Willd. & Er, Tr, NT & JAL 7830 \\
\hline \multicolumn{3}{|l|}{ APOCYNACEAE (L.S.Kinoshita) } \\
\hline Asclepias curassavica $\mathrm{L}$. & Er, Te, NT & MNS 6 \\
\hline Secondatia densiflora A.DC. & Arb, Tr, NT & JAL 7900 \\
\hline \multicolumn{3}{|l|}{ ARACEAE (L.G.Temponi) } \\
\hline Anthurium scandens (Aubl.) Engl. & Er, Ep, NT & JAL 7792 \\
\hline Anthurium sellowianum Kunth & Er, Ep, NT & JAL 7802, 8507 \\
\hline Asterostigma luschnatianum Schott & Er, Te, NT & JAL 7817 \\
\hline Heteropsis salicifolia Kunth & Er, Ep, NT & JAL 7793, 7976 \\
\hline Lemna valdiviana Phil. & $\mathrm{Er}, \mathrm{Aq}, \mathrm{NT}$ & JAL 8075 \\
\hline Philodendron appendiculatum Nadruz \& Mayo & Er, Ep, NT & JAL 7891 \\
\hline Philodendron martianum Engl. & Er, Ep, NT & JAL 7971 \\
\hline Philodendron propinquum Schott & Er, Ep, NT & JAL 7791 \\
\hline \multicolumn{3}{|l|}{ ARALIACEAE } \\
\hline Hydrocotyle leucocephala Cham. \& Schltdl. & Er, Te, NT & JAL 7915 \\
\hline \multicolumn{3}{|l|}{ ARECACEAE } \\
\hline Geonoma elegans Mart. & Arb, Te, NT & JAL 7800 \\
\hline Geonoma pohliana Mart. var. pohliana & Arb, Te, NT & JAL 7801 \\
\hline Geonoma pohliana var. rubescens (Wendland ex Drude) Henderson & Arb, Te, NT & JAL 7967 \\
\hline Lytocaryum hoehnei (Burret) Toledo & Arb, Te, NT & JAL 7856 \\
\hline \multicolumn{3}{|l|}{ ASTERACEAE (M.E.Monge) } \\
\hline Achyrocline satureioides (Lam.) DC. & Er, Te, NT & MNS 100 \\
\hline Ageratum conyzoides $\mathrm{L}$. & $\mathrm{Er}, \mathrm{Te}, \mathrm{NT}$ & MNS 20 \\
\hline
\end{tabular}




\begin{tabular}{|c|c|c|}
\hline Família/ Espécies & $\begin{array}{l}\text { Forma de vida, Hábito, } \\
\text { Categoria de ameaça }\end{array}$ & Coletor $n^{\circ}$ \\
\hline Baccharis anomala DC. & $\mathrm{Er}, \mathrm{Te}, \mathrm{NT}$ & MNS 112 \\
\hline Baccharis crispa Spreng. & $\mathrm{Er}, \mathrm{Te}, \mathrm{NT}$ & MNS 38 \\
\hline Baccharis dentata (Vell.) G.M.Barroso & $\mathrm{Er}, \mathrm{Te}, \mathrm{NT}$ & MNS 113 \\
\hline Bidens subalternus DC. & $\mathrm{Er}, \mathrm{Te}, \mathrm{NT}$ & MNS7; JAL7832 \\
\hline Chromolaena cf. laevigata (Lam.) R.M.King \& H.Rob. & $\mathrm{Er}, \mathrm{Te}, \mathrm{NT}$ & MNS 65 \\
\hline Conyza bonariensis (L.) Cronquist * & $\mathrm{Er}, \mathrm{Te}$ & MNS 10 \\
\hline Conyza sumatrensis (Retz) E.Walker * & $\mathrm{Er}, \mathrm{Te}$ & MNS 78 \\
\hline Cyrtocymura scorpioides (Lam.) H.Rob. & Er, Te, NT & MNS68;JAL7833 \\
\hline Erechtites valerianifolius (Wolf) DC. & $\mathrm{Er}, \mathrm{Te}, \mathrm{NT}$ & MNS 101 \\
\hline Gamochaeta coarctata (Willd.) Kerguélen & $\mathrm{Er}, \mathrm{Te}, \mathrm{NT}$ & JAL 7864 \\
\hline Jaegeria hirta (Lag.) Less. & $\mathrm{Er}, \mathrm{Te}, \mathrm{NT}$ & MNS 39 \\
\hline Leptostelma maxima D.Don & Er, Te, NT & JAL 8060 \\
\hline Mikania aff. cordifolia (L.f.) Willd. & Er, Tr, NT & MNS 64 \\
\hline Mikania hirsutissima DC. & $\mathrm{Er}, \mathrm{Tr}, \mathrm{NT}$ & MNS108 \\
\hline Mikania laevigata Sch.Bip. ex Baker & Er, Tr, NT & MNS81; JAL7857 \\
\hline Orthopappus angustifolius (Sw.) Gleason & $\mathrm{Er}, \mathrm{Te}, \mathrm{NT}$ & MNS 76 \\
\hline Piptocarpha notata (Less.) Baker & Arb, Tr, NT & JAL 8638 \\
\hline Exostigma notobellidiastrum (Griseb.) G.Sancho & Arb, Te, NT & MNS 122 \\
\hline Solidago chilensis Meyen & Er, Te, NT & MNS 55, 74 \\
\hline Sonchus oleraceus L. & $\mathrm{Er}, \mathrm{Te}, \mathrm{NT}$ & MNS 120 \\
\hline Sphagneticola trilobata (L.) Pruski & Er, Te, NT & JAL 7957 \\
\hline Vernonanthura petiolaris (DC.) H.Rob. & Er, Te, NT & MNS 73 \\
\hline Youngia japonica (L.) DC.* & $\mathrm{Er}, \mathrm{Te}$ & JAL 7865 \\
\hline Indeterminada 1 & $\mathrm{Er}, \mathrm{Te}$ & MNS 87 \\
\hline \multicolumn{3}{|l|}{ BALSAMINACEAE } \\
\hline Impatiens walleriana Hook.f.* & $\mathrm{Er}, \mathrm{Te}$ & MNS 52 \\
\hline \multicolumn{3}{|l|}{ BEGONIACEAE (L.Kollmann) } \\
\hline Begonia convolvulacea (Klotzsch) A.DC. & Er, Ep, NT & JAL 7807 \\
\hline Begonia fischeri Schrank & Er, Te, NT & MNS 102 \\
\hline Begonia fruticosa (Klotzsch) A.DC. & Er, Ep, NT & JAL 7816 \\
\hline Begonia semidigitata Brade & Arb, Te, NT & JAL 8538 \\
\hline \multicolumn{3}{|l|}{ BIGNONIACEAE } \\
\hline Amphilophium elongatum (Vahl) L.G.Lohmann & Arb, Tr, NT & JAL 7958 \\
\hline Mansoa difficilis (Cham.) Bureau \& K.Schum. & Arb, Tr, NT & MNS 106 \\
\hline \multicolumn{3}{|l|}{ BORAGINACEAE } \\
\hline Tournefortia bicolor Spreng. & Arb, Te, NT & JAL 8057 \\
\hline \multicolumn{3}{|l|}{ BROMELIACEAE (R.C.Forzza) } \\
\hline Aechmea gracilis Lindm. & $\mathrm{Er}, \mathrm{Ep}, \mathrm{VU}$ & JAL 7953 \\
\hline Billbergia amoena (Lodd.) Lindl. & Er, Ep, NT & JAL 8523 \\
\hline Billbergia distachia (Vell.) Mez. & Er, Ep, NT & MNS 107 \\
\hline
\end{tabular}




\begin{tabular}{|c|c|c|}
\hline Família/ Espécies & $\begin{array}{l}\text { Forma de vida, Hábito, } \\
\text { Categoria de ameaça }\end{array}$ & Coletor $\mathbf{n}^{\circ}$ \\
\hline Bromelia antiacantha Bertol. & Arb, Te, NT & JAL 7980 \\
\hline Nidularium cf. procerum Lindm. & Er, Te, NT & JAL 7846 \\
\hline Pseudananas sagenarius (Arruda) Camargo & Arb, Te, NT & JAL 7845 \\
\hline Tillandsia stricta Sol. & Er, Ep, NT & MNS 115 \\
\hline Tillandsia usneoides (L.) L. & Er, Ep, NT & JAL 7965 \\
\hline Vriesea carinata Wawra & Er, Ep, NT & MNS 1 \\
\hline Vriesea aff. flava A.F.Costa et al. & Er, Ep, NT & JAL 8519 \\
\hline Vriesea friburgensis Mez. & Er, Ep, NT & JAL 7932 \\
\hline Vriesea incurvata Gaudich. & Er, Ep, NT & JAL 7954 \\
\hline Vriesea philippocoburgii Wawra & Er, Ep, NT & JAL 8511 \\
\hline Vriesea procera (Mart. ex Schult \& Schult.f.) Wittm. & Er, Ep, NT & JAL 8516 \\
\hline Wittrockia cyathiformis (Vell.) Leme & Er, Ep, NT & JAL 8518 \\
\hline \multicolumn{3}{|l|}{ CACTACEAE (J.A.Lombardi) } \\
\hline Hatiora salicornoides (Haw.) Britton \& Rose & Er, Ep, NT & JAL 7896 \\
\hline Lepismium houlletianum (Lem.) Barthlott & Er, Ep, NT & JAL 7956 \\
\hline Rhipsalis burchellii Britton \& Rose & Er, Ep, NT & JAL7806, 7924 \\
\hline Rhipsalis floccosa Salm-Dyck ex Pfeiff. subsp. floccosa & Er, Ep, NT & JAL 7798 \\
\hline Rhipsalis oblonga Loefgr. & Er, Ep, NT & JAL8510, 8547 \\
\hline Rhipsalis teres Steud. & Er, Ep, NT & JAL 8062 \\
\hline \multicolumn{3}{|l|}{ CANNACEAE } \\
\hline Canna paniculata Ruiz \& Pav. & Er, Te, NT & MNS 21 \\
\hline \multicolumn{3}{|l|}{ CARYOPHYLLACEAE } \\
\hline Drymaria cordata (L.) Willd. ex Roem. \& Schult. & Er, Te, NT & MNS 43 \\
\hline \multicolumn{3}{|l|}{ COMMELINACEAE (L.Y.S.Aona) } \\
\hline Commelina diffusa Burm.f. & Er, Te, NT & MNS 119 \\
\hline Commelina obliqua Vahl & Er, Te, NT & MNS 69 \\
\hline Commelina rufipes var. glabrata (D.R.Hunt) Faden \& D.R.Hunt & $\mathrm{Er}, \mathrm{Te}, \mathrm{NT}$ & JAL 8044 \\
\hline Dichorisandra thyrsiflora J.C.Mikan & Er, Te, NT & JAL 7930 \\
\hline Dichorisandra villosula Mart. ex Schult \& Schult.f. & Er, Te, NT & JAL 7978 \\
\hline Tripogandra diuretica (Mart.) Handlos & Er, Te, NT & MNS 50 \\
\hline \multicolumn{3}{|l|}{ CONVOLVULACEAE (R.S.Bianchini) } \\
\hline Ipomoea cairica (L.) Sweet. & Er, Tr, NT & MNS 50 \\
\hline Ipomoea indica (Burm.f.) Merr. & Er, Tr, NT & JAL 7899 \\
\hline Ipomoea triloba $\mathrm{L}$. & Er, Tr, NT & JAL 8524 \\
\hline \multicolumn{3}{|l|}{ COSTACEAE } \\
\hline Costus spiralis (Jacq.) Roscoe & Arb, Te, NT & MNS 103 \\
\hline \multicolumn{3}{|l|}{ CUCURBITACEAE } \\
\hline Cayaponia sp. & $\mathrm{Er}, \operatorname{Tr}$ & JAL 7882, 7929 \\
\hline Melothria sp. & $\mathrm{Er}, \operatorname{Tr}$ & JAL 7928 \\
\hline Sycios sp. & $\mathrm{Er}, \mathrm{Tr}$ & JAL 7975 \\
\hline
\end{tabular}




\begin{tabular}{|c|c|c|}
\hline Família/ Espécies & $\begin{array}{l}\text { Forma de vida, Hábito, } \\
\text { Categoria de ameaça }\end{array}$ & Coletor $n^{\circ}$ \\
\hline Willbrandia sp. & Er, $\operatorname{Tr}$ & JAL 8073 \\
\hline \multicolumn{3}{|l|}{ CYPERACEAE (M.Alves) } \\
\hline Carex seticulmis Boeckeler & Er, Te, NT & JAL 7838 \\
\hline Cyperus surinamensis Rottb. & $\mathrm{Er}, \mathrm{Te}, \mathrm{NT}$ & JAL 7938 \\
\hline Cyperus sp. & $\mathrm{Er}, \mathrm{Te}$ & MNS 71 \\
\hline Eleocharis sp. & $\mathrm{Er}, \mathrm{Aq}$ & JAL 8632 \\
\hline Hypolytrum schraderianum Nees & $\mathrm{Er}, \mathrm{Te}, \mathrm{NT}$ & MNS 128 \\
\hline Pleurostachys gaudichaudii Brogn. & $\mathrm{Er}, \mathrm{Te}, \mathrm{NT}$ & JAL 7837 \\
\hline Rhynchospora sp.1 & $\mathrm{Er}, \mathrm{Te}$ & JAL 7885 \\
\hline Rhynchospora sp.2 & Er, Te & JAL 8032 \\
\hline Scleria panicoides Kunth & $\mathrm{Er}, \mathrm{Te}, \mathrm{NT}$ & MNS 9 \\
\hline \multicolumn{3}{|l|}{ DILLENIACEAE } \\
\hline Davilla kunthii A.St.-Hil. & Arb, Tr, NT & MNS 67 \\
\hline \multicolumn{3}{|l|}{ DIOSCOREACEAE (D. Araújo) } \\
\hline Dioscorea monadelpha (Kunth) Griseb. & Er, Tr, NT & JAL 7906 \\
\hline \multicolumn{3}{|l|}{ EUPHORBIACEAE } \\
\hline Croton fuscescens Spreng. & Er, Te, NT & JAL 7823 \\
\hline Croton lundianus (Didr.) Müll.Arg. & Er, Te, NT & MNS 90 \\
\hline Croton sp. & Er, Te & JAL 7941 \\
\hline Dalechampia cf. scandens L. & Er, Tr, NT & JAL 7826 \\
\hline \multicolumn{3}{|l|}{ FABACEAE (A.M.A.G.Tozzi) } \\
\hline Canavalia sp. & $\mathrm{Er}, \mathrm{Tr}$ & MNS 117 \\
\hline Crotalaria breviflora DC. & Er, Te, NT & MNS 49 \\
\hline Dalbergia frutescens (Vell.) Britton var. frutescens & Er, Tr, NT & JAL 7963 \\
\hline Desmodium adscendens (Sw.) DC. & Er, Te, NT & JAL 8064 \\
\hline Desmodium uncinatum (Jacq.) DC. & Er, Te, NT & JAL 8040 \\
\hline \multicolumn{3}{|l|}{ GENTIANACEAE } \\
\hline Voyria aphylla (Jacq.) Pers. & Er, Sap, NT & JAL 8033 \\
\hline \multicolumn{3}{|l|}{ GESNERIACEAE } \\
\hline Nematanthus fritschii Hoehne & Er, Ep, NT & MNS 58 \\
\hline Nematanthus monanthos (Vell.) Chautems & Er, Ep, VU & MNS 57 \\
\hline Sinningia douglasii (Lindl.) Chautems & Er, Ep, NT & JAL 7912 \\
\hline \multicolumn{3}{|l|}{ HELICONIACEAE } \\
\hline Heliconia sp.1 & $\mathrm{Er}, \mathrm{Te}$ & JAL 7879 \\
\hline Heliconia sp.2 & $\mathrm{Er}, \mathrm{Te}$ & JAL 7799 \\
\hline \multicolumn{3}{|l|}{ HYPOXIDACEAE } \\
\hline Curculigo capitulata Kuntze* & $\mathrm{Er}, \mathrm{Te}$ & JAL 7894 \\
\hline Hypoxis decumbens $\mathrm{L}$. & Er, Te, NT & MNS 45 \\
\hline \multicolumn{3}{|l|}{ IRIDACEAE } \\
\hline Sisyrinchium micranthum Cav. & Er, Te, NT & JAL 7862, 7878 \\
\hline
\end{tabular}




\begin{tabular}{|c|c|c|}
\hline Família/ Espécies & $\begin{array}{l}\text { Forma de vida, Hábito, } \\
\text { Categoria de ameaça }\end{array}$ & Coletor $n^{\circ}$ \\
\hline Trimezia sp. & Er, Te & JAL 8066 \\
\hline \multicolumn{3}{|l|}{ JUNCACEAE } \\
\hline Juncus capillaceus Lam. & Er, Te, NT & JAL 7960 \\
\hline Juncus cf. micranthus Schr. ex E.Meyer & Er, Te, NT & JAL 7959 \\
\hline \multicolumn{3}{|l|}{ LAMIACEAE } \\
\hline Hyptis recurvata Poit. & Er, Te, NT & JAL 7870 \\
\hline Hyptis sp. & Er, Te & MNS 37 \\
\hline Ocimum selloi Benth. * & $\mathrm{Er}, \mathrm{Te}$ & MNS 47 \\
\hline \multicolumn{3}{|l|}{ LAXMANIACEAE } \\
\hline Cordyline fruticosa (L.) A.Chev.* & Er, Te & MNS 26 \\
\hline \multicolumn{3}{|l|}{ LOASACEAE } \\
\hline Blumenbachia scabra (Miers) Urb. & Er, Tr, NT & JAL 8631 \\
\hline \multicolumn{3}{|l|}{ LOGANIACEAE } \\
\hline Spigelia beyrichiana Cham. \& Schltdl. & $\mathrm{Er}, \mathrm{Te}, \mathrm{NT}$ & JAL 7895,7872 \\
\hline Spigelia pusilla Mart. & Er, Te, NT & JAL 7913 \\
\hline \multicolumn{3}{|l|}{ LORANTHACEAE } \\
\hline Struthanthus concinnus Mart. & Arb, HPar, NT & MNS 2 \\
\hline Struthanthus cf. flexicaulis (Mart.) Mart. & Arb, HPar, NT & JAL 7892 \\
\hline \multicolumn{3}{|l|}{ LYTHRACEAE } \\
\hline Cuphea calophylla subsp. mesostemom (Koehne) Lourteig & Er, Te, NT & MNS 40 \\
\hline \multicolumn{3}{|l|}{ MALPIGHIACEAE (A.M.A.Amorim) } \\
\hline Heteropterys nitida (Lam.) Humb., Bonpl. \& Kunth & Arb, Tr, NT & JAL 8050 \\
\hline \multicolumn{3}{|l|}{ MALVACEAE } \\
\hline Sida rhombifolia $\mathrm{L}$. & Er, Te, NT & MNS 89 \\
\hline Sida sp. & Arb, Te & JAL 8067 \\
\hline Pavonia communis A.St.-Hil. & Er, Te, NT & MNS 75 \\
\hline Triumfetta semitriloba Jacq. & Er, Te, NT & MNS 34 \\
\hline \multicolumn{3}{|l|}{ MARANTACEAE } \\
\hline Ctenanthe kummeriana (E.Morren) Eichler & $\mathrm{Er}, \mathrm{Te}, \mathrm{NT}$ & JAL 8054 \\
\hline Ctenanthe lanceolata Petersen & Er, Te, NT & JAL 7977 \\
\hline Goeppertia monophylla (Vell.) Borchs. \& S.Suárez & Er, Te, NT & JAL 8068 \\
\hline Goeppertia sp. & Er, Te & JAL 7931 \\
\hline Stromanthe papillosa Petersen & Er, Te, NT & JAL 7955 \\
\hline \multicolumn{3}{|l|}{ MARCGRAVIACEAE } \\
\hline Marcgravia polyantha Delp. & Arb, Tr, NT & JAL 7907 \\
\hline \multicolumn{3}{|c|}{ MELASTOMATACEAE (R.Romero, R.Goldenberg \& F.S.Meyer) } \\
\hline Bertolonia mosenii Cogn. & Er, Te, NT & JAL7803, 7947 \\
\hline Clidemia hirta (L.) D.Don & Er, Te, NT & MNS 85 \\
\hline Leandra aurea (Cham.) Cogn. & Arb, Te, NT & MNS 70 \\
\hline Leandra cf. carassana (DC.) Cogn. & Arb, Te, NT & MNS 18 \\
\hline
\end{tabular}




\begin{tabular}{|c|c|c|}
\hline Família/ Espécies & $\begin{array}{l}\text { Forma de vida, Hábito, } \\
\text { Categoria de ameaça }\end{array}$ & Coletor $n^{\circ}$ \\
\hline Leandra cardiophylla Cogn. & $\mathrm{Arb}, \mathrm{Te}, \mathrm{NT}$ & JAL 7836 \\
\hline Leandra dasytricha (A.Gray) Cogn. & Arb, Te, NT & MNS 17 \\
\hline Leandra fragilis Cogn. & Arb, Te, NT & MNS123 \\
\hline Leandra laevigata (Triana) Cogn. & $\mathrm{Arb}, \mathrm{Te}, \mathrm{NT}$ & JAL 7855, 7944 \\
\hline Leandra melastomoides Raddi & Arb, Te, NT & MNS 14 \\
\hline Leandra nianga (DC.) Cogn & Arb, Te, NT & MNS125 \\
\hline Leandra regnellii (Triana) Cogn. & Arb, Te, NT & JAL 7946 \\
\hline Leandra xanthocoma (Naudin) Cogn. & Arb, Te, NT & JAL 7942 \\
\hline Pleiochiton blepharodes (DC.) Reginato et al. & Er, Ep, NT & MNS 56 \\
\hline Tibouchina cerastiifolia (Naud.) Cogn. & $\mathrm{Er}, \mathrm{Te}, \mathrm{NT}$ & MNS 118 \\
\hline Tibouchina clinopodifolia (DC.) Cogn. & $\mathrm{Er}, \mathrm{Te}, \mathrm{NT}$ & MNS 88 \\
\hline Tibouchina sp. & Er, Te & JAL 7973 \\
\hline \multicolumn{3}{|l|}{ MENISPERMACEAE } \\
\hline Cissampelos andromorpha DC. & Er, Tr, NT & JAL 7903 \\
\hline \multicolumn{3}{|l|}{ OCHNACEAE } \\
\hline Sauvagesia erecta $\mathrm{L}$. & $\mathrm{Er}, \mathrm{Te}, \mathrm{NT}$ & JAL 8031 \\
\hline \multicolumn{3}{|l|}{ ONAGRACEAE } \\
\hline Fuchsia regia (Vell.) Munz & Arb, Te, NT & MNS 121 \\
\hline Ludwigia elegans (Cambess.) H.Hara & Er, Te, NT & JAL 7925 \\
\hline \multicolumn{3}{|l|}{ ORCHIDACEAE (W.Forster) } \\
\hline Anathallis microgemma (Schltr. ex Hoehne) Pridgeon \& M.W.Chase & Er, Ep, NT & JAL 7840 \\
\hline Aspidogyne hylibates (Rchb.f.) Garay & Er, Te, NT & JAL 8039 \\
\hline Baskervilla paranaensis (Kraenzl.) Schltr. & Er, Ep, NT & MNS 91 \\
\hline Bifrenaria aureofulva Lindl. & Er, Ep, NT & JAL 8070 \\
\hline Brasiliorchis chrysantha (Barb.Rodr.) R.B.Singer, S.Koehler \& Carnevali & Er, Ep, NT & JAL 7911 \\
\hline Campylocentrum aromaticum Barb.Rodr. & Er, Ep, NT & JAL 8515 \\
\hline Campylocentrum micranthum (Lindl.) Rolfe & Er, Ep, NT & JAL 8042 \\
\hline Coppensia flexuosa (Sims) Campacci & Er, Ep, NT & JAL 7974 \\
\hline Eurystyles actinosophila (Barb.Rodr.) Schltr. & Er, Ep, NT & JAL 8049 \\
\hline Gomesa crispa (Lindl.) Klotzsch \& Rchb.f. & Er, Ep, NT & MNS 60 \\
\hline Leptotes bicolor Lindl. & Er, Ep, NT & JAL 7841 \\
\hline Liparis nervosa (Thunb.) Lindl. & Er, Ep, NT & JAL 8956 \\
\hline Malaxis excavata (Lindl.) Kuntze & Er, Te, NT & JAL 8525 \\
\hline Octomeria gracilis Lodd. ex Lindl. & Er, Ep, NT & JAL 7839 \\
\hline Octomeria praestans Lindl. & Er, Ep, NT & JAL 7819 \\
\hline Phymatidium delicatulum Lindl. & Er, Ep, NT & JAL 7935 \\
\hline Polystachya micrantha Schltr. & Er, Ep, NT & JAL 8069 \\
\hline Prescottia densiflora (Brogn.) Cogn. & Er, Te, NT & JAL 7860 \\
\hline Prescottia stachyodes (Sw.) Lindl. & Er, Te, NT & JAL 7851, 8526 \\
\hline Prosthechea glumacea (Lindl.) W.E.Higgins & $\mathrm{Er}, \mathrm{Ep}, \mathrm{NT}$ & JAL 8034 \\
\hline
\end{tabular}




\begin{tabular}{|c|c|c|}
\hline Família/ Espécies & $\begin{array}{l}\text { Forma de vida, Hábito, } \\
\text { Categoria de ameaça }\end{array}$ & Coletor $n^{\circ}$ \\
\hline Rodriguezia bracteata (Vell.) Hoehne & Er, Ep, NT & JAL 7936 \\
\hline Trizeuxis falcata Lindl. & Er, Ep, NT & JAL 7881 \\
\hline Zygopetalum maxillare Lodd. & Er, Ep, VU & JAL 8038 \\
\hline \multicolumn{3}{|l|}{ OROBANCHACEAE } \\
\hline Castilleja arvensis Cham. \& Schltdl. & $\mathrm{Er}, \mathrm{Te}, \mathrm{NT}$ & JAL 7861 \\
\hline \multicolumn{3}{|l|}{ PASSIFLORACEAE (D.A.Araújo) } \\
\hline Passiflora haematostigma Mart. ex. Mast. & $\mathrm{Er}, \mathrm{Tr}, \mathrm{NT}$ & JAL 7825 \\
\hline Passiflora misera Kunth & Er, Tr, NT & JAL 8528 \\
\hline \multicolumn{3}{|l|}{ PIPERACEAE (E.F.Guimarães) } \\
\hline Peperomia catharinae Miq. & Er, Ep, NT & JAL 8036 \\
\hline Peperomia hispidula (Sw.) A.Dietr. & $\mathrm{Er}, \mathrm{Te}, \mathrm{NT}$ & JAL7815, 7952 \\
\hline Peperomia martiana Miq. & Er, Ep, NT & JAL 8035 \\
\hline Peperomia urocarpa Fisch. \& C.A.Mey & Er, Ep, NT & JAL 7852, 8074 \\
\hline Piper aduncum L. & Arb, Te, NT & MNS 23 \\
\hline Piper caldense C.DC. & Arb, Te, NT & JAL 7789 \\
\hline Piper cernuum Vell. & Arb, Te, NT & JAL 8503 \\
\hline Piper lucaeanum var. grandifolium Yunck. & Arb, Te, NT & JAL 7950 \\
\hline Piper miquelianum C.DC. & Arb, Te, NT & MNS 13 \\
\hline Piper reitzii Yunck. & Arb, Te, NT & MNS 93 \\
\hline Piper rivinoides Kunth & Arb, Te, NT & MNS 22 \\
\hline Piper setebarraense E.F.Guim. \& L.H.P.Costa & Arb, Te, NT & JAL 8029 \\
\hline Piper solmsianum C.DC. & Arb, Te, NT & JAL 7890 \\
\hline Piper umbellatum $\mathrm{L}$. & Arb, Te, NT & MNS 35,83 \\
\hline Piper sp. & Arb, Te & JAL 7811 \\
\hline \multicolumn{3}{|l|}{ PLANTAGINACEAE } \\
\hline Plantago australis Lam. * & $\mathrm{Er}, \mathrm{Te}$ & JAL 7858 \\
\hline Scoparia dulcis L. & $\mathrm{Er}, \mathrm{Te}, \mathrm{NT}$ & MNS 82 \\
\hline \multicolumn{3}{|c|}{ POACEAE (R.T.Shirasuna, T.S.Filgueiras \& R.S.Rodrigues) } \\
\hline Andropogon bicornis L. & Er, Te, NT & MNS 79 \\
\hline Axonopus polystochyus G.A.Black & $\mathrm{Er}, \mathrm{Te}, \mathrm{NT}$ & JAL 8041 \\
\hline Chusquea ramosissima Lindm. & Er, Tr, NT & JAL 8543 \\
\hline Ichnanthus leiocarpus (Spreng.) Kunth & $\mathrm{Er}, \mathrm{Te}, \mathrm{NT}$ & JAL 7968 \\
\hline Melinis minutiflora P.Beauv.* & $\mathrm{Er}, \mathrm{Te}$ & MNS 48, 66 \\
\hline Ocellochloa stolonifera (Poir.) Zuloaga \& Morrone & $\mathrm{Er}, \mathrm{Te}, \mathrm{NT}$ & JAL 7970 \\
\hline Olyra glaberrima Raddi & Er, Te, NT & JAL 7951 \\
\hline Panicum pilosum $\mathrm{Sw}$. & $\mathrm{Er}, \mathrm{Te}, \mathrm{NT}$ & MNS 96 \\
\hline Parodiolyra micrantha (Kunth) Davidse \& Zuloaga & Er, Te, NT & JAL 7854, 7888 \\
\hline Paspalum corcovadense Raddi & $\mathrm{Er}, \mathrm{Te}, \mathrm{NT}$ & JAL 7969 \\
\hline Paspalum conjugatum Berg. & $\mathrm{Er}, \mathrm{Te}, \mathrm{NT}$ & JAL 8058 \\
\hline Pharus lappulaceus Aubl. & $\mathrm{Er}, \mathrm{Te}, \mathrm{NT}$ & JAL 8045 \\
\hline
\end{tabular}




\begin{tabular}{|c|c|c|}
\hline Família/ Espécies & $\begin{array}{l}\text { Forma de vida, Hábito, } \\
\text { Categoria de ameaça }\end{array}$ & Coletor $n^{\circ}$ \\
\hline Steinchisma laxa (Sw.) Zuloaga & Er, Te, NT & JAL 7945 \\
\hline Urochloa brizantha (Hochst. ex A.Rich.) Webster* & $\mathrm{Er}, \mathrm{Te}$ & MNS 12 \\
\hline \multicolumn{3}{|l|}{ POLYGALACEAE } \\
\hline Polygala lancifolia A.St.-Hil. \& Moq. & Er, Te, NT & MNS 84,JAL 7943 \\
\hline \multicolumn{3}{|l|}{ POLYGONACEAE } \\
\hline Polygonum acuminatum Kunth & Er, Te, NT & JAL 8051 \\
\hline Polygonum meisnerianum Cham. \& Schltdl. & Er, Te, NT & JAL 8514 \\
\hline \multicolumn{3}{|l|}{ PORTULACACEAE } \\
\hline Talinum paniculatum (Jacq.) Gaertn. & Er, Te, NT & JAL 7979 \\
\hline \multicolumn{3}{|l|}{ ROSACEAE } \\
\hline Rubus rosifolius $\mathrm{Sm}$. & Arb, Te, NT & MNS 53 \\
\hline Rubus sellowii Cham. \& Schltdl. & Arb, Te, NT & MNS 41 \\
\hline \multicolumn{3}{|l|}{ RUBIACEAE (P.Delprete) } \\
\hline Coccocypselum hasslerianum Chodat & $\mathrm{Er}, \mathrm{Te}, \mathrm{NT}$ & JAL7818, 8513 \\
\hline Coccocypselum lymansmithii Standl. & Er, Te, NT & MNS 59 \\
\hline Faramea cf. latifolia (Cham. \& Schltdl.) DC. & Arb, Te, NT & JAL 7834, 7909 \\
\hline Galium hypocarpium (L.) Endl. ex Griseb. & $\mathrm{Er}, \mathrm{Te}, \mathrm{NT}$ & MNS 124 \\
\hline Manettia congesta (Vell.) K.Schum. & Er, Tr, NT & JAL 8520 \\
\hline Manettia gracilis Cham. \& Schltdl. & $\mathrm{Er}, \mathrm{Tr}, \mathrm{NT}$ & MNS 92 \\
\hline Manettia paraguariensis Chodat & Er, Tr, NT & MNS 51 \\
\hline Palicourea croceoides Ham. & Arb, Te, NT & MNS 29 \\
\hline Psychotria racemosa Rich. & Arb, Te, EX & JAL 8506 \\
\hline Psychotria rueliifolia (Cham. \& Schltdl.) Müll.Arg. & $\mathrm{Er}, \mathrm{Te}, \mathrm{NT}$ & MNS 28 \\
\hline Psychotria suterella Müll.Arg. & Arb, Te, NT & MNS 3 \\
\hline Randia armata (Sw.) DC. & Arb, Te, NT & JAL 7850 \\
\hline Spermacoce verticillata $\mathrm{L}$. & $\mathrm{Er}, \mathrm{Te}, \mathrm{NT}$ & MNS 44 \\
\hline \multicolumn{3}{|l|}{ SAPINDACEAE } \\
\hline Paullinia carpopoda Cambess. & Er, Tr, NT & MNS19 \\
\hline Paullinia paradoxa Radlk. & Er, Tr, NT & JAL 7828 \\
\hline Paullinia seminuda Radlk. & Er, Tr, NT & JAL 8635 \\
\hline \multicolumn{3}{|l|}{ SMILACACEAE } \\
\hline Smilax remotinervis Hand.-Mazz. & Er, Tr, NT & JAL 7902 \\
\hline \multicolumn{3}{|l|}{ SOLANACEAE (J.R.Stehmann \& L.L.Giacomin) } \\
\hline Aureliana fasciculata (Vell.) Sendtn. & Arb, Te, NT & JAL 7916 \\
\hline Brunfelsia uniflora (Pohl) D.Don & Arb, Te, NT & JAL 7898 \\
\hline Brunfelsia cuneifolia J.A.Schmidt & Arb, Te, NT & JAL 7871 \\
\hline Capsicum flexuosum Sendtn. & Arb, Te, NT & JAL 7972 \\
\hline Cestrum bracteatum Link \& Otto & Arb, Te, NT & MNS 95 \\
\hline Dyssochroma viridiflorum (Sims) Miers & Arb, Ep, NT & JAL8541, 8639 \\
\hline Solanum americanum Mill. & Arb, Te, NT & MNS 86 \\
\hline
\end{tabular}




\begin{tabular}{|c|c|c|}
\hline Família/ Espécies & $\begin{array}{l}\text { Forma de vida, Hábito, } \\
\text { Categoria de ameaça }\end{array}$ & Coletor $n^{\circ}$ \\
\hline Solanum argenteum Dunal & $\mathrm{Arb}, \mathrm{Te}, \mathrm{NT}$ & JAL 7859 \\
\hline Solanum concinnum Schott. ex Sendtn. & Arb, Te, NT & MNS 105 \\
\hline Solanum granulosoleprosum Dunal & $\mathrm{Arb}, \mathrm{Te}, \mathrm{NT}$ & JAL7824, 8517 \\
\hline Solanum hoehnei C.V.Morton & Er, Te, NT & JAL 7869, 7937 \\
\hline Solanum pseudocapsicum L. & $\mathrm{Arb}, \mathrm{Te}, \mathrm{NT}$ & JAL 7887 \\
\hline Solanum swartzianum Roem. \& Schult. & Arb, Te, NT & MNS 63 \\
\hline Solanum variabile Mart. & Arb, Te, NT & JAL 7886 \\
\hline \multicolumn{3}{|l|}{ TRIGONIACEAE } \\
\hline Trigonia nivea Cambess. & Er, Tr, NT & JAL 7926 \\
\hline \multicolumn{3}{|l|}{ URTICACEAE } \\
\hline Boehmeria caudata Sw. & Er, Te, NT & JAL 7961 \\
\hline Pilea nummulariifolia (Sw.) Wedd.* & $\mathrm{Er}, \mathrm{Te}$ & JAL 8028 \\
\hline Urera nitida (Vell.) P.Brack & Arb, Te, NT & MNS 27, 62 \\
\hline \multicolumn{3}{|l|}{ VALERIANACEAE } \\
\hline Valeriana scandens Loefl. & $\mathrm{Er}, \mathrm{Tr}, \mathrm{NT}$ & JAL 7849 \\
\hline \multicolumn{3}{|l|}{ VERBENACEAE } \\
\hline Lantana undulata Schrank & Er, Te, NT & MNS 72 \\
\hline Stachytarpheta cayennensis (Rich.) Vahl & Arb, Te, NT & MNS 104 \\
\hline Verbena $\mathrm{cf}$. litoralis Kunth & Er, Te, NT & JAL 7962 \\
\hline \multicolumn{3}{|l|}{ VIOLACEAE } \\
\hline Anchietea pyrifolia (Mart.) G.Don & $\mathrm{Er}, \mathrm{Tr}, \mathrm{NT}$ & JAL 7829 \\
\hline \multicolumn{3}{|l|}{ VITACEAE (J.A.Lombardi) } \\
\hline Cissus sulcicaulis (Baker) Planch. & Arb, Tr, NT & JAL 7940 \\
\hline Cissus verticillata (L.) Nicolson \& C.E.Jarvis subsp. verticillata & Arb, Tr, NT & JAL 8542 \\
\hline \multicolumn{3}{|l|}{ ZINGIBERACEAE } \\
\hline Hedychium coronarium J.Koenig* & $\mathrm{Er}, \mathrm{Te}$ & MNS 5 \\
\hline
\end{tabular}

Forma de vida/ Hábito/ Categoria de Ameaça: Er - Herbáceo, Arb - Arbustivo, Te - Terrícola, Ep - Epífita, Tr - Trepador, Aq - Aquático, HPar - Hemiparasita, Sap - Saprófita, NT - Não ameaçada, VU - Vulnerável, EX - presumivelmente extinta. * indica espécies não nativas da flora brasileira. Especialistas consultados em parênteses após a respectiva família.

Life form/ Habit/ Threatened Category: Er - Herbaceous, Arb - Shrub, Te - Terrestrial, Ep - Epiphyte, Tr - Climber, Aq - Aquatic, HPar - Hemi-parasite, Sap - Saprophyte, NT - not threatened, VU - vulnerable, EX - presumable extinct. * indicates species not native to the brazilian flora. Specialists who contribute with identification are cited between parenthesis after the respective family.

\section{Conclusão}

A alta riqueza e composição de espécies não arbóreas na RPPN Paiol Maria reafirma que a diversidade de espécies em pequenos fragmentos deve ser levada em conta em planos de conservação (Gentry 1992), uma vez que estas áreas podem manter elevado número de espécies e consequentemente grande número de relações ecológicas, imprescindíveis para o funcionamento do ecossistema (Brown \& Lugo 1990).
A supressão de vegetação primária faz com que florestas secundárias atuem como refúgio de espécies (Chazdón et al. 2009) e, neste sentido, a baixa similaridade da composição de espécies epífitas entre áreas de Floresta Ombrófila Densa Montana da Serra do Mar ressalta a importância da conservação todos os fragmentos, independente de tamanho ou localização (Menini Neto et al. 2009). Fragmentos mais antigos e próximos de áreas protegidas podem ser fundamentais no 
caso da execução de planos de manejo visando a implantação de corredores ecológicos ou reintrodução de espécies.

\section{Agradecimentos}

Aos especialistas, as identificações (indicados entre parênteses após respectiva família); ao $\mathrm{CNPq}$, a bolsa de Mestrado concedida à primeira autora (processo $\mathrm{n}^{\circ}$ 130726/2010-9); ao MCT/ $\mathrm{CNPq} / \mathrm{MEC} / \mathrm{CAPES}$, a concessão de auxílio ao projeto do segundo autor (PROTAX processo $\mathrm{n}^{\circ}$ 562240/2010-1); aos revisores e ao Editor de área, as sugestões e contribuições ao trabalho; e à Vitae Civilis, a permissão da realização deste estudo.

\section{Referências}

Aguiar, A.P.; Chiarello, A.G.; Mendes, S.L. \& Matos, E.N. 2003. The Central and Serra do Mar Corridors in the Atlantic Forest of Brazil. In: Galindo-Leal, C. \& Câmara, I.B. (eds.). The Atlantic Forest of South America: biodiversity status, threats, and outlook. Island Press, Washington. Pp. 118-132.

APG III. 2009. An update of the Angiosperm Phylogeny Group classification for the orders and families of flowering plants: APG III. Botanical Journal of the Linnean Society 161: 105-121.

Barlow, J.; Gardner, T.A.; Araujo, I.S.; Ávila-Pires, T.C.; Bonaldo, A.B.; Costa, J.E.; Esposito, M.C.; Ferreira, L.V.; Hawes, J.; Hernandez, M.I.M.; Hoogmoed, M.S.; Leite, R.N.; Lo-Man-Hung, N.F.; Malcolm, J.R.; Martins, M.B.; Mestre, L.A.M.; Miranda-Santos, R.; Nunes-Gutjahr, A.L.; Overal, W.L.; Parry, L.; Peters, S.L.; Ribeiro-Junior, M.A.; Silva, M.N.F.; Silva-Motta, C. \& Peres, C.A. 2007. Quantifying the biodiversity value of tropical primary, secondary and plantation forests. PNAS 104: 18555-18560.

BFG - The Brazil Flora Group. 2015. Growing knowledge: an overview of Seed Plant diversity in Brazil. Rodriguésia (online) 66: 1085-1113.

Blum, C.T.; Roderjan, C.V. \& Galvão, F. 2011. Composição florística e distribuição altitudinal de epífitas vasculares da Floresta Ombrófila Densa na Serra da Prata, Morretes, Paraná, Brasil. Biota Neotropica 11: 141-159.

Brown, S. \& Lugo, A.E. 1990. Tropical secondary forests. Journal of Tropical Ecology 6: 1-32.

Carnaval, A.N.; Hickerson, M.J.; Haddad, C.F.B.; Rodrigues, M.T. \& Moritz, C. 2009. Stability predicts genetic diversity in the Brazilian Atlantic Forest hotspot. Science 323: 785-789.

CEPAGRI - Centro de Pesquisas Metereológicas e Climáticas relacionadas à agricultura, UNICAMP. 2011. Clima dos Municípios Paulistas. Disponível em
$<$ http://www.cpa.unicamp.br/outras-informacoes/ clima-dos-municipios-paulistas.html>. Acesso em 13 dezembro 2011.

Chazdón, R.L.; Peres, C.A.; Dent, D.; Sheil, D.; Lugo, A.E.; Lamb, D.; Stork, N.E. \& Miller, S.E. 2009. The potential for species conservation in tropical secondary forests. Conservation Biology 23: 14061417.

Dislich, R. \& Mantovani, W. 1998. A flora de epífitas vasculares da Reserva da Cidade Universitária "Armando Salles Oliveira" (São Paulo, Brasil). Boletim de Botânica da Universidade de São Paulo 17: 61-83.

Filgueiras, T.S.; Nogueira, P.E.; Brochado, A.L. \& Guala II, G.F. 1994. Caminhamento - um método expedito para levantamentos florísticos qualitativos. Cadernos de Geociências 12: 39-43.

Galindo-Leal, C. \& Câmara, I.G. 2005. Status do hotspot Mata Atlântica: uma síntese. In: Mata Atlântica: biodiversidade, ameaças e perspectivas. Fundação SOS Mata Atlântica, São Paulo. Pp. 3-9.

Garcia, R.J.F. \& Pirani, J.R. 2005. Análise florística, ecológica e fitogeográfica do Núcleo Curucutu, Parque Estadual da Serra do Mar (São Paulo, SP), com ênfase nos campos junto à crista da Serra do Mar. Hoehnea 32: 1-48.

Gentry, A.H. 1992. Tropical forest biodiversity: distributional patterns and their conservational significance. Oikos 63: 19-28.

Gilliam, F.S. 2007. The ecological significance of the herbaceous layer in temperate forest ecosystems. BioScience 57: 845-858.

Gonçalves, E.G. 2012. Asterostigma Fisch. \& C.A.Mey. In: Wanderley, M.G.L.; Shepherd, G.J.; Melhem, T.S.; Giulietti, A.M. \& Martins, S.E. Flora Fanerogâmica do Estado de São Paulo. Instituto de Botânica, São Paulo. Vol. 7, pp. 46-47.

Groppo, M. \& Pirani, J.R. 2005. Levantamento florístico das espécies de ervas, subarbustos, lianas e hemiepífitas da mata da reserva da cidade universitária "Armando Salles Oliveira”, São Paulo, SP, Brasil. Boletim de Botânica da Universidade de São Paulo 23: 141-233.

Hogan, D.J.; Carmo, R.L.; Rodrigues, I.A. \& Alves, H.P.F. 2000. Conflitos entre crescimento populacional e uso dos recursos ambientais em bacias hidrográficas do estado de São Paulo. In: TORRES, H. \& COSTA, H. (org.). População e Meio Ambiente - Debates e Desafios. Ed. SENAC, São Paulo. Pp. 233-269.

IBGE (Instituto Brasileiro de Geografia e Estatística). 2002. Perfil dos Municípios Brasileiros - Meio Ambiente. Instituto Brasileiro de Geografia e Estatística, São Paulo. 388p.

IBGE (Instituto Brasileiro de Geografia e Estatística). 2012. Manual Técnico da Vegetação Brasileira. $2^{\text {a }}$ ed. Instituto Brasileiro de Geografia e Estatística, Rio de Janeiro. 272p. 
Kramer, K.U. \& Tryon, R.M. 1990. Introduction to the treatment of pteridophytes. In: Kramer, K.U. \& Green, P.S (eds.). The families and genera of vascular plants - I. Pteridophytes and Gymnosperms. Springer-Verlag, Berlin. Pp. 12-13.

Lees, A. \& Peres, C.A. 2008. Conservation value of remnant riparian forest corridors of varying quality for Amazonian birds and mammals. Conservation Biology 22: 439-449.

Liesbsch, D.; Goldenberg, R. \& Marques, M.C.M. 2007. Florística e comunidades vegetais emu ma cronosequênca de Floresta Atlântica no Estado do Paraná, Brasil. Acta Botanica Brasilica 21: 983-992.

Lima, R.A.F.; Dittrich, V.A.O.; Souza, V.C.; Salino, A.; Breier, T.B. \& Aguiar, O.T. 2011. Flora vascular do Parque Estadual Carlos Botelho, São Paulo, Brasil. Biota Neotropica 11: 1-9.

Mamede, M.C.H.; Souza, V.C.; Prado, J.; Barros, F.; Wanderley, M.G.L. \& Rando, J.G. (org.). 2007. Livro Vermelho das Espécies Vegetais Ameaçadas do Estado de São Paulo. Instituto de Botânica, São Paulo. 165p.

Menini Neto, L.; Forzza, R.C. \& Zappi, D. 2009. Angiosperm epiphytes as conservation indicators in forest fragments: A case study from southeastern Minas Gerais, Brazil. Biodiversity Conservation 18: 3785-3807.

Mittermeier, R.A.; Gil, P.R.; Hoffmann, M.; Pilgrim, J.; Brooks, T.; Mittermeier, C.G.; Lamoreux, J. \& Fonseca, G.A.B. 2005. Hotspots Revisited: Earth's Biologically Richest and Most Endangered Terrestrial Ecoregions. Conservation International. 392p.

Morellato, L.P.C. \& Haddad, C.F.B. 2000. The Brazilian Atlantic Forest. Biotropica 32: 786-792.

Mori, S.A.; Mattos-Silva, L.A.; Lisboa, G. \& Coradin, L. 1989. Manual de manejo do herbário fanerogâmico, $2^{\mathrm{a}}$ ed. Centro de Pesquisas do Cacau, Ilhéus. 104p.

Mueller-Dombois, D. \& Ellenberg, H. 1974. Aims and Methods of Vegetation Ecology. $2^{\text {nd }}$ ed. John Wiley \& Sons, New York. 531p.

Neiman, Z.; Cardoso-Leite, E. \& Podadera, D.S. 2009. Planejamento e implantação participativos de programa de interpretação em trilhas na "RPPN Paiol Maria", Vale do Ribeira (SP). Revista Brasileira de Ecoturismo 2: 11-34.

Oliveira-Filho, A.T; Carvalho, D.A.; Vilela, E.A.; Curi, N. \& Fontes, M.A.L. 2004. Diversity and structure of the tree community of a fragment of tropical secondary forest of the Brazilian Atlantic Forest domain 15 and 40 years after logging. Revista Brasileira de Botânica 27: 685-701.

Pavan-Fruehalf, S. 2000. Plantas medicinais da Mata Atlântica - Manejo Sustentado e Amostragem. Ed. Fapesp, São Paulo. 216p.

Ribeiro, M.C.; Metzger, J.P.; Martensen, A.C.; Ponzoni, F.J. \& Hirota, M.M. 2009. The Brazilian Atlantic Forest: how much is left, and how is the remaining forest distributed? Implications for conservation. Biological Conservation 142: 1141-1153.

Richards, P.W. 1996. The tropical rain forest: an ecological study. $2^{\text {nd }} E d$, Cambridge University Press, Cambridge. 600p.

São Paulo (Estado) - Departamento de Águas e Energia Elétrica. 1966. Serviço do Vale do Ribeira. Plano de Desenvolvimento do Vale do Ribeira e Litoral Sul. Vol. 1. Brasconsult, São Paulo. Pp. 27-33.

Shepherd, G.J. 2009. FITOPAC 2.1 (versão preliminar). Departamento de Biologia Vegetal, Universidade Estadual de Campinas, Campinas. Disponível em $<$ http://pedroeisenlohr.webnode.com.br/fitopac/>. Acesso em 13 dezembro 2011.

Silva, J.M.C. \& Casteleti, C.H.M. 2005. Estado da biodiversidade da Mata Atlântica brasileira. In: GALINDO-LEAL, C. \& CÂMARA, I.G. (eds.). Mata Atlântica: Biodiversidade, Ameaças e Perspectivas. Fundação SOS Mata Atlântica, São Paulo. Pp. 43-59.

Smith, A.R.; Pryer, K.M.; Schuettpelz, E.; Korall, P.; Schneider, H. \& Wolf, P.G. 2006. A classification for extant ferns. Taxon 55: 705-731.

Spyreas, G. \& Matthews, J.W. 2006. Floristic conservation value, nested understory floras, and the development of second-growth forest. Ecological Applications 16: 1351-1366.

Tabarelli, M.; Aguiar, A.V.; Ribeiro, M.C.; Metzger, J.P. \& Peres, C.A. 2010. Prospects for biodiversity conservation in the Atlantic Forest: lessons from aging human-modified landscapes. Biological Conservation 143: 2328-2340.

VITAE CIVILIS - Instituto para Desenvolvimento, Meio Ambiente e Paz (ONG). Reserva Particular Paiol Maria. Disponível em: $<$ http://www.ecoturismosls. org.br/default.asp? siteAcao=mostraPagina\&pagina $\mathrm{Id}=87>$. Acesso em 31 janeiro 2012.

Werneck, M.S.; Sobral, M.E.G.; Rocha, C.T.V.; Landau, E.C. \& Stehmann, J.R. 2011. Distribution and endemism of angiosperm in the Atlantic Forest. Natureza \& Conservação 9: 188-193.

Wright, S.J. \& Muller-Landau, H.C. 2006. The future of tropical forest species. Biotropica 38: 287-301. 
